\section{$\underset{\substack{\text { hommes } \\ \text { \& migrations }}}{ }$}

\section{Hommes \& migrations}

Revue française de référence sur les dynamiques

migratoires

\section{$1299 \mid 2012$}

Musulmanes et féministes en Grande-Bretagne

\title{
Pakistan. Le féminisme réinventé
}

\section{Afiya Shehrbano Zia}

Traducteur : Marie-Hélène Dumas

\section{(2) OpenEdition}

Journals

Édition électronique

URL : http://journals.openedition.org/hommesmigrations/1854

DOI : 10.4000/hommesmigrations. 1854

ISSN : 2262-3353

Éditeur

Musée national de l'histoire de l'immigration

Édition imprimée

Date de publication : 1 septembre 2012

Pagination : 86-95

ISSN : 1142-852X

Référence électronique

Afiya Shehrbano Zia, "Pakistan. Le féminisme réinventé », Hommes \& migrations [En ligne], 1299 | 2012, mis en ligne le 31 décembre 2014, consulté le 30 avril 2019. URL : http://

journals.openedition.org/hommesmigrations/1854; DOI : 10.4000/hommesmigrations.1854 


\section{Pakistan. Le féminisme réinventé(1)}

Par Afiya Shehrbano Zia, chercheure, Lyceum College, Karachi

Pour les femmes musulmanes, la pratique de la religion offre un refuge face au pouvoir patriarcal des hommes. Au Pakistan, pays d'origine de nombreuses femmes installées en Grande-Bretagne, le mouvement des femmes laïques, en s'alliant avec les mouvements de femmes islamistes, s'est vu absorbé par ces derniers. Aujourd'hui, les féministes musulmanes constituent un mouvement diversifié et s'engagent de plus en plus. 
Prétendre que les événements du 11 septembre ont créé de "nouvelles" identités religieuses pour les femmes du Pakistan serait une erreur. La "guerre mondiale contre la terreur" a bien joué sur les identités politiques des femmes en produisant une opposition plus marquée entre celles qui seraient perçues comme des Pakistanaises libérales, modernes, et les Pakistanaises talibanisées, régressives et conservatrices. Pourtant, les débats qui ont lieu au sein des mouvements des femmes pakistanaises montrent qu'il ne faut pas y déceler le résultat du 11 septembre mais qu'au contraire, la palette des diverses tendances féministes se situe dans la continuité du passé.

Dans la mesure où les deux stéréotypes mentionnés ci-dessus sont construits à partir d'un discours patriarcal plus général sur le mouvement des femmes et les identités nationalistes, il serait plus exact de dire que l'identité politique de la femme pakistanaise est le résultat d'une coalescence plutôt que d'une renaissance. Ce qui est nouveau, c'est l'acceptation générale d'une opposition Occident/ Islam plus claire dans le Pakistan de l'après-11 septembre, et des défis politiques qui en découlent. Dans ce cadre, les identités des femmes musulmanes semblent bien être devenues un sujet de recherche intéressant - un nouveau terrain d'études.

Les femmes continuent en même temps d'être utilisées comme de puissants symboles du renouveau mondial des mouvements islamiques. La notion de "capacité d'action" des femmes musulmanes, et sa récupération par les nationalismes religieux "masculinistes" (si bien analysée dans les études du féminisme postcolonial) est maintenant revisitée dans les arènes politiques locales et internationales de l'après- 11 septembre. Le symbolisme de la "femme musulmane" est tout autant instrumentalisé par les critiques postcoloniales des Lumières que par les gouvernements des sociétés musulmanes qui cherchent à émousser l'islamisme radical et par les islamistes euxmêmes.

Qu'il s'agisse du voile, des crimes d'honneur ou même de l'occupation des espaces publics ou de la possibilité de se déplacer, le corps de la femme musulmane est devenu plus qu'un signifiant du nationalisme ou qu'un marqueur de l'islam contre le discours occidental (colonisateur). Il est devenu la bannière que brandissent les critiques postcoloniales musulmanes pour défendre la "capacité d'agir dans la docilité" des femmes pratiquantes ou croyantes ${ }^{(2)}$. Dans le contexte musulman, même les interprétations religieuses locales rivalisent quant au potentiel symbolique de l'être-femme musulmane. Les groupes fondamentalistes et extrémistes sont de plus en plus conscients du pouvoir symbolique de "la femme musulmane" et prêts à le manipuler.

Cet article se propose de démontrer que le nouvel intérêt porté à la femme musulmane remonte à une querelle qui a déjà hanté les militantes féministes urbaines du Pakistan 
se battant pour les droits des femmes. Savoir si elles devaient agir à l'intérieur de cadres laïcs ou islamiques est une question qui s'est posée bien avant le 11 septembre. Les nouveaux débats théoriques sur la "capacité d'action" de la femme islamiste ont cependant permis à des groupes religieux masculinistes rivaux de s'emparer de la question pour élaborer leur nouvelle politique, littéralement à travers le corps des femmes, se rendant compte du potentiel constitué par cette notion de la femme musulmane silencieuse mais dotée de pouvoir sur sa propre vie. La "guerre mondiale contre la terreur" n'a fait que prolonger les clivages préexistants et apporter crédibilité et légitimité politiques au féminisme religieux comme alternative à une défense des droits des femmes plus générale, occidentalisée, impérialiste et sponsorisée par les États-Unis.

D'autre part, nous voyons les islamistes et les femmes pratiquantes prendre des positions de contestation politique. Aujourd'hui, la religion n'est plus autant un "pont entre le domaine spirituel et le domaine social ${ }^{3 /}$ " mais davantage un lien avec le domaine politique. Ce qui discrédite l'idée selon laquelle l'expression politique de la religion serait extérieure aux objectifs personnels et à la capacité d'agir des musulmanes pakistanaises en fonction de leur confession religieuse.

La vision romanesque d'une musulmane pratiquante autonome a largement permis à l'islam de se déployer pour "libéraliser ou réguler la politique du corps des femmes"(4)", beaucoup plus que l'on ne pouvait s'y attendre dans le contexte pakistanais. Pour la première fois dans l'interprétation des extrémistes islamiques non gouvernementaux du Pakistan, la mise en ceuvre des idéologies extrémistes a inclu les femmes : pour atteindre les buts qu'ils visaient, les activistes se sont appropriés la capacité d'agir militante des musulmanes. En conséquence, des attentats suicides ont été commis par des femmes et des châtiments sévères ont été infligés à celles qui résistent aux extrémistes, et qui sont donc considérées comme des infidèles qui trahissent la cause de l'islam ${ }^{(5)}$.

\section{Contexte historique de la lutte pour les droits des femmes au Pakistan}

C'est sous la dictature éclairée du général Ayub Khan (1958-1969) que la première vague de militantes féministes pakistanaises, en particulier l'Association des femmes de tout le Pakistan (APWA) fit campagne contre les Lois de la famille musulmane sur les droits à l'héritage et au divorce des femmes, et sur la restriction de la polygamie. C'est ainsi qu'en 1961, l'ordonnance sur les lois de la famille musulmane (MFLO) fut décrétée et légalement mise en ceuvre ${ }^{(6)}$. 
Pour de nombreuses militantes de l'ancienne génération, cette époque demeure "l'âge d'or". Avec le recul, on se rend compte de l'importance symbolique de leur action en ce qu'elle a rendu les identités et les droits des femmes pakistanaises très différents de ceux des femmes des pays arabes à majorité musulmane.

En 1977, l'armée prend le pouvoir et, pendant la décennie suivante, sous le joug du général Zia-ul-Haq's et sous prétexte d'islamisation du pays, les femmes sont devenues la cible d'un État misogyne ${ }^{(7)}$. Un des legs les plus importants de cette période troublée est que l'analyse de la situation des femmes au Pakistan ne peut se décliner qu'à l'aune d'une politique confessionnelle et de la résistance ou de l'obéissance des femmes aux conséquences de cette politique. Après avoir retracé les diverses tendances du mouvement des femmes qui nous ont amenées là, il s'agit de démontrer que les influences géopolitiques telles que la modernité et la globalisation ne sont qu'une partie de ce que les femmes doivent consciemment aborder, négocier ou affronter. Plus encore, cet article met en Les féminismes
pakistanais sont depuis
des années aux prises
avec des identités
polarisées et fragmentées
liées aux identités
religieuses. avant le fait que l'élaboration d'un nouveau féminisme et de nouvelles relations entre les femmes et l'État ont été réalisables grâce à une double action de résistance aux idéaux libéraux (et modernes) et leur récupération par les femmes des mouvements fondamentalistes islamiques de droite. Au cours de ce processus, les objectifs aussi bien que les méthodes du mouvement des femmes pakistanaises progressistes ont été remis en cause, et, dans ce contexte, le féminisme a dû être redéfini.

Une telle perspective historique est importante en ce qu'elle permet de comprendre que l'émergence de ces identités ne peut pas être exclusivement attribuée à une influence occidentale externe, ni être seulement considérée comme le résultat de l'impérialisme ou de la mondialisation. Le féminisme pakistanais est depuis des années aux prises avec des identités polarisées et fragmentées liées aux identités religieuses. La proposition centrale de cet article est que les stratégies productrices

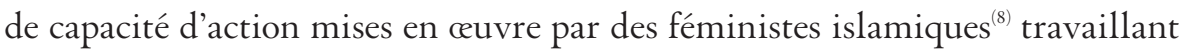
dans le cadre de la religion n'ont pas seulement été couronnées de succès, elles ont fini par subsumer toutes les autres formes de féminismes.

Par son approche des questions religieuses de façon inclusive et bienveillante, le mouvement des femmes a involontairement permis et favorisé le féminisme islamique tel qu'il est aujourd'hui. 


\section{La question de l'utilisation stratégique des droits islamiques}

C'est au sein du Forum d'action des femmes (WAF), alliance formée en 1981 autour de groupes et d'individus se battant contre l'islamisation et la loi martiale, qu'on s'est le plus ouvertement demandé s'il fallait défendre les droits des femmes de l'intérieur de la posture islamique ou dans un cadre laïque.

Le mouvement des femmes, y compris le WAF, décida tout d'abord d'utiliser les interprétations progressistes de l'islam afin de contrer la religion d'État patriarcale ${ }^{(9)}$. Jusqu'à un certain point, cette stratégie a réussi au mouvement et, chose importante, elle lui a valu, contre toute attente, le soutien de femmes islamistes de droite comme celui de modérées - tout au moins d'un point de vue stratégique. C'était un soutien imprévisible car le mouvement des femmes urbaines incluait plusieurs représentantes de partis politiques progressistes, d'idéologies de gauche et de groupements professionnels tels que des syndicats ou des groupes traitant la question du développement. Au sujet du viol, par exemple, les femmes des partis fondamentalistes de droite participaient aux manifestations contre l'État, mais tout en prenant leurs distances avec les slogans du WAF contre les ordonnances Hudood ou l'islamisation ${ }^{(10)}$. En 1991, après des années de discussion, le WAF s'écarta radicalement de son histoire féministe libérale et se déclara laïque - en principe. Cette décision fit cependant naître une série de nouveaux débats à l'intérieur du mouvement dans son ensemble, débats dont on peut dire qu'ils restent à ce jour irrésolus.

À la lumière de cette expérience historique, Saba Mahmood, professeur et critique des lumières postcoloniales et de la laïcité libérale, signale une rupture intéressante en se référant à son bref engagement personnel dans le mouvement féministe progressiste pakistanais. Elle rappelle que pendant les années d'islamisation, les militantes considéraient les formes islamiques de patriarcat comme les racines de l'oppression des femmes, et que "la politique féministe en arriva à exiger une position laïque résolue et inflexible ${ }^{(11) "}$.

L'engagement de l'auteure du présent article dans le WAF de Lahore et sa participation aux discussions qui animèrent le mouvement dans les années quatre-vingt-dix laissent penser que cette position n'était pas aussi catégorique, et que tout en demeurant inflexible sur le principe, cette position est toujours restée ouverte à la négociation et à la réflexion stratégique.

Les féministes islamiques modernistes ${ }^{(12)}$ reprochent, entre autres, à la position laïque de ne pas avoir encouragé la mobilisation des femmes de toutes les classes sociales et pensent que, étant donné le débat chronophage qui l'a entourée, elle n'a peut-être pas été le meilleur choix ${ }^{(13)}$. Elles avancent qu'au lieu de "se lancer tête baissée dans la 
confrontation, il aurait peut-être mieux valu établir des distinctions entre les différentes tendances religieuses, traditionnelles et culturelles ${ }^{(14)}$," cette posture libérale s'oppose au choix prescrit entre identité religieuse et identité laïque respectivement imposées aux femmes par les forces politico-religieuses et les "groupes féministes dominants".

Selon moi, tel est l'espace exact dans lequel le mouvement s'est retrouvé prêt à être récupéré et absorbé par une force qui le dépassait, plus puissante et plus gratifiante que les groupes de femmes progressistes ne pouvaient l'être, que ce soit sur le plan personnel ou politique, ou sur celui de la sororité spirituelle. Pendant les années quatre-vingt, les services secrets pakistanais, financés par le gouvernement américain, créèrent une force de guérilla idéologique et armée (les talibans) afin de lutter contre l'invasion communiste de l'Afghanistan ${ }^{(15)}$. Presque parallèlement, certaines sections du mouvement des femmes pakistanaises participèrent à la création d'un féminisme alternatif, un féminisme islamique, instrument de lutte contre la religion de l'État patriarcal. Il ne faut peut-être pas attribuer à celles qui défendaient les droits des femmes dans un esprit de progrès la responsabilité d'avoir recruté des militantes du mouvement fondamentaliste islamique et de les avoir financées. Mais la stratégie des alliances, qui a fait appel au point de vue religieux et s'en est accommodée, a cependant accordé une légitimité de féministes à celles qui travaillaient de l'intérieur de la parole islamique, sans tenir compte du cadre patriarcal qui définissait et restreignait cette parole.

Sans nier la capacité d'action des femmes de la droite religieuse, il faut souligner que leur position a toujours critiqué et repoussé le féminisme libéral moderne occidental ${ }^{(16)}$. L'État et certaines sections du mouvement des femmes ont utilisé l'islam comme une arme de défense politique contre les idéologies "étrangères", l'un contre le communisme, les autres contre le féminisme occidental. Ce qui a doté de capacité d'agir et de crédibilité les nouveaux mouvements religieux. Un phénomène qu'illustre particulièrement bien l'attitude des militantes islamistes de la madrassa Jamia Hafsa, qui se sont opposées avec force au régime de Gen Musharraf lors du soulèvement de la mosquée Lal Masjid en $2007^{(17)}$.

La structure, la nature et le bien-fondé du WAF ont été l'objet de nombreux débats et discussions au sein du mouvement des femmes pakistanaises urbaines, principalement du fait que l'on compte parmi ses membres des théoriciennes, des universitaires et des professionnelles du développement aptes à l'analyse et dont les carrières dépendent de leurs recherches sur la question des femmes. Leurs différents points de vue, en particulier en ce qui concerne la direction que doivent suivre les militantes, et le cadre dans lequel elles doivent le faire, sont souvent contestés, récusés, critiqués par des membres mêmes du WAF, qui y voient "trop de réflexion et pas assez d'action". La plupart du temps, tout dialogue interactif sur ces questions se trouve interrompu, sans 
qu'on soit arrivé à une conclusion, car nourrir des débats sur la question des femmes est considéré par beaucoup comme une tâche intellectuelle, trop éloignée des problèmes de développement ou des préoccupations pratiques en général.

En fait, le mouvement des femmes, quelle que soit la définition qu'on en donne, se scinde et part dans de nouvelles directions aussi inattendues qu'intéressantes.

\section{Défis analytiques}

L'analyse classique de l'oppression des femmes, qui a vu le jour au Pakistan dans les années quatre-vingt, tendait à reposer sur l'hypothèse selon laquelle les identités religieuses contemporaines et la conscientisation liée à la foi étaient directement héritées de la dictature du général Zia. Ce qui s'oppose à toutes les recherches sur l'expérience postcoloniale de la construction des identités, selon lesquelles les différents acteurs et personnalités des États postcoloniaux ont à la longue mis en déroute la définition de tradition et de modernité, afin de restaurer, construire et nourrir de nouvelles identités politiques et religieuses ${ }^{(18)}$. Persister à faire de toutes les identités religieuses actuelles le produit de l'islamisation du général Zia a conduit à ce sur quoi Amina Jamal ${ }^{(19)}$ et de nombreux autres chercheurs attirent l'attention : le refus de reconnaître la capacité d'agir et l'autonomie des forces islamistes de droite, et en particulier des femmes qui appartiennent à ces mouvements.

Depuis, une génération d'intellectuels pakistanais post-11 septembre étudie, révise, redéfinit l'histoire économique, sociale et juridique de l'islam, afin d'interpréter de façon inédite les mouvements sociopolitiques du monde musulman. C'est une génération pour qui la métaphore de l'islamisation oppressive du général Zia n'est rien en comparaison de la colère ressentie vis-à-vis de l'islamophobie occidentale associée à la persécution anglo-américaine des musulmans dans le monde entier.

Si une part du militantisme qui s'est développé dans la dernière décennie peut de toute évidence être perçue comme s'inscrivant dans la continuation idéologique et matérielle de l'État instauré par le général Zia, il existe d'autres influences productrices elles aussi de nouvelles identités religieuses. Parmi elles, notons "l'arabisation" croissante des cultures locales due aux migrations des travailleurs vers les pays du Golfe, ainsi que le développement des groupes confessionnels de femmes et des rites et pratiques religieux privés.La religion a pris un nouvel essor après le 11 septembre, quand les femmes ont cherché une nouvelle forme d'expression politique au sein de la résistance à l'islamophobie définie par les hommes.

De nombreuses jeunes femmes, en particulier celles des classes moyennes inférieures, trouvèrent refuge dans la religion face à une société qui leur déniait tout pouvoir sur 
leur vie et où elles avaient de moins en moins de droits et étaient de moins en moins représentées. On peut en voir un exemple chez celles qui se mirent à porter le hijab en tant que symbole religieux, et qui y virent ensuite une protection contre le harcèlement sexuel ainsi qu'une façon d'obtenir plus d'espace dans la sphère publique. En prêchant chez elles, certaines se sentirent investies de pouvoir sur leurs petites cours, les dars (groupes d'études confessionnels), et elles compensèrent ainsi l'impuissance qui était la leur au sein de la démocratie comme dans leur vie privée. Lectures du Coran et deuils individuels sont de plus en plus souvent remplacés par les pratiques des dars.

La capacité d'agir politique des islamistes s'étend maintenant bien au-delà de simples attitudes religieuses ou d'une interprétation dite erronée de la religion. Ce mouvement jouit dans bien des cas d'un soutien populaire sincère et c'est par les services qu'il rend, la puissance d'agir qu'il confère et la justice sociale qu'il apporte qu'il a réussi à institutionnaliser sa cause. Les normes qui encadrent cette religiosité revivifiée sont toujours discriminatoires par rapport aux femmes et aux minorités. Ce qui ne veut pas dire qu'elles s'expriment toujours sous une forme violente. Elles sont au contraire insidieusement introduites à travers les ceuvres caritatives.

De nombreuses initiatives du militantisme religieux se cachent derrière des organiDe nombreuses jeunes
femmes, en particulier celles
des classes moyennes
inférieures, trouvèrent refuge
dans la religion face à
une société qui leur déniait,
par ailleurs, tout pouvoir
sur leur vie et où elles avaient
de moins en moins
de droits et étaient de moins
en moins représentées. sations de développement. Elles relèvent souvent de la même loi et réunissent des fonds en incluant les droits des femmes dans leur raison d'être et leurs objectifs. Non contentes d'imiter ce qu'elles percevaient comme la recette efficace du militantisme féministe laïque, les militantes des groupes les plus à droite se confrontent à l'État de plus en plus souvent et de façon de plus en plus radicale. Elles y réussissent parce qu'elles se savent plus nombreuses et plus puissantes que les féministes laïques. En s'accommodant d'un "féminisme culturel musulman" apparemment apolitique et en réussissant à remettre en cause le patriarcat à partir d'interprétations progressistes de l'islam, elles ont suivi une stratégie qui connaît un certain succès. Elles ont donc appris la stratégie politique, elles n'en aspirent pas moins à transformer l'État en une véritable nation islamique fonctionnant selon la charia.

Parallèlement à l'activisme croissant des femmes islamistes, le cyber féminisme joue un rôle mineur, plus fluide et plus virtuel que réel, mais grâce au développement des nouveaux médias, beaucoup de jeunes ont accès aux tribunes libres des journaux et 
peuvent donner leur avis d'internautes à la télévision. Cela bien qu'ils aient rarement directement coopéré avec les mouvements populaires et qu'ils ne se soient jamais politiquement confrontés à l'État.

Une nouvelle campagne contre les ordonnances Hudood, comme en a mené le mouvement des femmes depuis vingt-cinq ans, a peu de chances de voir le jour. Exactement de la même façon que, sur le plan local, la justice tribale "règle" les disputes, l'État a résolu les problèmes des femmes grâce à de légères modifications de la loi sans en questionner le fondement ni la raison d'être. Il est peu probable qu'un autre gouvernement ait suffisamment de pouvoir, ou de soutien de la part des libéraux, pour faire disparaître les lois discriminatoires, à partir d'une position de principe ou du point de vue d'un impératif international.

\section{Un nouveau féminisme?}

D'un point de vue historique, prétendre qu'agir dans le cadre de l'islam a aidé à toucher les différentes classes sociales et à réduire les contradictions de classe est faux. L'organisation d'obédience musulmane Al-Huda tolère les femmes qui choisissent de ne pas porter le voile lors des réunions religieuses ou dans les écoles ${ }^{(20)}$; Jamia Hafsa exige que les femmes portent le voile intégral noir. Aucun critère ni symbole n'est exigé de celles qui veulent appartenir au mouvement libéral des femmes. Chacune peut changer d'opinion, que ce soit sur le plan politique ou personnel, quel que soit le sujet et quelle que soit l'époque, et continuer d'en faire idéologiquement partie de façon permanente. Les sociologues pourraient rejeter, à un certain niveau, les fausses oppositions entre modérées, libérales et extrémistes et mettre en avant qu'elles appartiennent toutes au même éventail social. Les féministes se sont toujours réjouies de la diversité et de la pluralité au sein de leur mouvement où, des libérales aux islamiques, toutes les tendances sont représentées. Les leçons de l'histoire devraient cependant nous apprendre que, de façon récurrente, les espaces conçus pour accueillir les expressions culturelles de la religion ont, au Pakistan, tout bonnement éclipsé, nié et délégitimé le féminisme progressif.

Il n'y a pas si longtemps, en 2002, la militante pour les droits des femmes Farida Shaheed, de tendance islamiste moderne, s'opposait aux militantes urbaines qui, avançait-elle, ont dominé la pensée du mouvement. Elle leur reproche de vouloir réprimer les pratiques de "contestation culturelle", y compris sur la base de la religion". Elle souligne le besoin de reconnaître que la religion opère simultanément comme une quête personnelle spirituelle permettant à chacune d'agir sur sa propre vie et comme une force mobilisatrice qui contredit parfois la doctrine religieuse. Elle 
critique en ce sens les militantes des classes moyennes férues de théorie féministe qui devraient, dit-elle, se méfier de leur propre "présupposition" lorsqu'elles remettent en cause les interprétations féministes de l'islam. Elle nous met en garde contre toute contestation de ces projets révisionnistes qui contredisent, au sein du mouvement, la position féministe laïque.

Les pratiques productrices de capacité d'agir dont elle vante le succès, telles que les dars, les khatams (prières pour les morts) et autres usages religieux définissant des relations sociales, sont précisément celles qui ont abouti à la politisation de la religion et aux répercussions sociales dont nous avons parlé plus haut. Shaheed souligne combien il est important que les femmes expriment à leur façon leur rapport à la religion. Mahmood conteste l'idée que les modes de sociabilité définis par celles qui choisissent de se conduire "de manière islamique" en opposition aux attitudes "occidentales libérales", puissent être "apolitiques par leur nature même $e^{(22) " . ~ E l l e ~ a v a n c e ~ q u ' a u ~ c o n t r a i r e, ~ p r e ́ t e n d r e ~}$ que "les mouvements liés à la foi ne confrontent pas l'État directement ${ }^{23) " ~ e s t ~ u n e ~ e r r e u r . ~ E n ~}$ effet, cet appel politique à la religion est le produit de la "présupposition" qui a conduit les féministes islamiques modernistes à croire en un avenir politique progressif au sein d'une culture et d'une religion qu'elles pensaient pouvoir redéfinir. Quelles qu'aient été leurs hypothèses, elles sont aujourd'hui incapables d'arrêter la dynamique politique réactionnaire de ces mouvements.

Il est très probable que le mouvement des femmes pakistanaises se concentrera désormais avant tout sur la participation à la vie politique, mais pas nécessairement sur la capacité d'action personnelle; il se centrera sur la violence contre les femmes, mais en mettant l'accent sur les victimes plutôt que sur la prévention; et il ne réussira à remettre en question ni les réseaux de négociation informelle entre hommes, ni les pratiques culturelles misogynes. Et surtout, il est presque certain que nous allons assister à une opposition croissante entre "bonnes et mauvaises musulmanes", où celles qui respectent une interprétation libérale de la théologie se trouveront confrontées à celles qui suivent un mode d'interprétation strict et littéral des textes religieux et s'associent à la pensée politico-religieuse masculiniste ${ }^{(24)}$.

\section{Traduit de l’anglais par Marie-Hélène Dumas}

\section{Notes}

\footnotetext{
* Note de la traductrice : Les termes "agency" et "empowerment", largement utilisés dans la théorie féministe anglo-saxonne, n’ont pas de véritables équivalents français. Sont utilisées les traductions les plus courantes : "capacité d'action" et "puissance d'agir", avec parfois la notion d'autonomie pour "agency" et de "pouvoir sur sa propre vie" pour "empowerment". Le mot "masculiniste" a été conservé, peu courant en français, mais construit dans le jargon anglo-saxon de la théorie du genre comme le pendant de "féministe", signifiant donc "défendant les droits des hommes". "Secular",
} 
qui signifie non religieux ou dépourvu de religion, a été traduit par le terme "laïque", bien que ce soit une spécificité française (entre autres) et que cette réalité ne trouve pas son équivalent exact au Pakistan.

1. Une version corrigée de cet article est déjà parue sous le titre "The reinvention of feminism in Pakistan" in Feminist Review, n 91, pp. 29-46, 2009. Bien des sujets traités ici sont aussi développés dans un essai intitulé "Motivated by dictatorship, muted by democracy", in Voicing Demands, Londres, Zed Books, à paraître, 2012.

2. Saba Mahmood, Politics of Piety : the Islamic Revival and the Feminist Subject, Princeton, NJ, Princeton University Press, 2005.

3. Farida Shaheed, "Women's Experiences of Identity, Religion and Activism in Pakistan", in Syed Naseem et Khalid Nadvi (dir.), The Post-Colonial State and Social Transformation in India and Pakistan, Karachi, Oxford University Press, 2002, p. 366.

4. Aihwa Ong, "State Versus Islam: Malay families, Women's bodies and the Body Politic in Malaysia", in Reina Lewis and Sara Mills (dir.), Feminist Postcolonial Theory: a Reader, New York, Routledge, 2003, pp. 381-412.

5. Afiya Zia, "Female suicide bombings in Pakistan; what's in it for women?", in OpenDemocracy, 4 October 2011, www.opendemocracy.net/5050/afiya-shehrbano-zia/female-suicide-bombings-in-pakistan-whats-in-it-for-women.

6. Karin Carmet Yefet, "The Constitution and Female-Initiated Divorce in Pakistan: Western Liberalism in Islamic Garb", in Harvard Journal of Law and Gender, n 34, pp. 553-615. Yefet montre à quel point cette ordonnance était hardie.

7. Asma Jahangir et H. Jilani, The Hudood Ordinances : A Divine Sanction?, Lahore, Rohtas Books. 1990 ; Afiya Zia, Sex Crime in the Islamic Context ; Rape, Class and Gender in Pakistan, Lahore, ASR Publications, 1994.

8. J'utilise ce terme pour désigner les féministes qui cherchent à développer la capacité d'action des femmes à l'intérieur d'un "islam repensé" et travaillent à réinterpréter et réexaminer la lecture masculiniste du Coran et de la charia. Et en particulier à deux universitaires pakistanaises (de la disapora): Riffat Hasan et Asma Barlas.

9. Nighat Said Khan, Voices Within: Dialogues with Women on Islam, Lahore, ASR, 1992.

10. Ces lois, passées en 1979, faisaient partie de la politique d'islamisation du général Zia-ul-Haq. L'ordonnance Zina, qui a fait de l'adultère un crime contre l'État passible de la peine de mort, brouille la frontière entre viol et rapports consentis. Des centaines et des centaines de femmes, victimes de cette loi, se sont retrouvées en prison. Elle a été amendée en 2006/2007, en accord avec l'Acte de protection des femmes.

11. Saba Mahmood, op. cit., p. $x$.

12. Certaines chercheures musulmanes modernistes, comme Azza Karam, utilisent l'expression "féministes islamiques" afin de distinguer féministes et non-féministes au sein d'un militantisme islamique qui les englobe.

Voir Tahmina Rashid, Contested Representation : Punjabi Women in Feminist Debate in Pakistan, Karachi, Oxford University Press, 2006, p. 71. Cette distinction est également reconnue et utilisée à l'intérieur du mouvement des femmes pakistanaises.

13. Farida Shaheed, op. cit., p. 373.

14. Ibid., p. 381 .

15. Ahmed Rashid, Taliban : Islam, Oil and the New Great Game in Central Asia, Londres and New York, I.B. Tauris, 2000.

16. Tahmina Rashid, op.cit.

17. Afiya Zia, "Faith-Based Politics, Enlightened Moderation and the Pakistani Women's Movement", in Journal of International Women's Studies (Internet edition), vol. 11, n 1, 2009. www.bridgew.edu/SoAS/JIWS/Nov09/Afiya.pdf

18. Homi Bhabha, Nation and Narration, London, New York, Routledge, 1990.

19. Amina Jamal, "Feminist 'Selves' and Feminism's 'Others'; Feminist Representations of Jamaat-e-Islami Women in Pakistan", in Feminist Review, n 81, 2005, pp. 52-73.

20. Sadaf Ahmad, Transforming Faith; The Story of Al-Huda and Islamic Revivalism Among Urban Pakistani Women, New York, Syracuse University Press, 2009.

21. Farida Shaheed, op. cit.

22. Saba Mahmood, Politics of Piety: the Islamic Revival and the Feminist Subject, op. cit., p. 73.

23. Ibid. 